OPEN

SUBJECT AREAS:

ELECTRONIC PROPERTIES

AND MATERIALS

LASER-PRODUCED PLASMAS

Received

19 February 2014

Accepted

16 April 2014

Published

14 May 2014

Correspondence and requests for materials should be addressed to

F.B. (filippo.

bencivenga@elettra.

eu) or C.M. (claudio.

masciovecchio@

elettra.eu)

\section{Reflectivity enhancement in titanium by ultrafast XUV irradiation}

\author{
F. Bencivenga' ${ }^{1}$, E. Principi ${ }^{1}$, E. Giangrisostomi ${ }^{1,2}$, R. Cucini ${ }^{1}$, A. Battistoni ${ }^{1}, 2$, F. D'Amico ${ }^{1}$, A. Di Cicco ${ }^{3}$, S. Di \\ Fonzo' ${ }^{1}$ A. Filipponi ${ }^{4}$, A. Gessini' ${ }^{1}$ R. Gunnella ${ }^{3}$, M. Marsi ${ }^{5}$, L. Properzi ${ }^{3}$, M. Saito' \& C. Masciovecchio'
}

'Elettra-Sincrotrone Trieste S.C.p.A., S.S. 14 km 163, 5 in AREA Science Park, 1-34149 Basovizza, Italy, ${ }^{2}$ Dipartimento di Fisica, Università degli Studi di Trieste, 34127 Trieste, Italy, ${ }^{3}$ Physics Division, School of Science and Technology, Università di Camerino, I-62032 Camerino (MC), Italy, ${ }^{4}$ Dipartimento di Scienze Fisiche e Chimiche, Università degli Studi dell'Aquila, I-67100 L'Aquila, Italy, ${ }^{5}$ Laboratoire de Physique des Solides, CNRS-UMR 8502, Université Paris-Sud, FR-91 405 Orsay, France.

The study of highly photo-excited matter at solid state density is an emerging field of research, which is benefitting the development of free-electron-laser (FEL) technology. We report an extreme ultraviolet (XUV) reflectivity experiment from a titanium (Ti) sample irradiated with ultrafast seeded FEL pulses at variable incident photon fluence and frequency. Using a Drude formalism we relate the observed increase in reflectivity as a function of the excitation fluence to an increase in the plasma frequency, which allows us to estimate the free electron density in the excited sample. The extreme simplicity of the experimental setup makes the present approach potentially a valuable complementary tool to determine the average ionization state of the excited sample, information of primary relevance for understanding the physics of matter under extreme conditions.

r

he study of matter under extreme thermodynamic conditions is a longstanding and fruitful field of research that has generated a host of applications in fundamental physics and applied cutting-edge technology ${ }^{1,2}$. In the last few decades increasing efforts have been devoted to studying new states of matter such as strongly coupled plasma and warm dense matter $(\mathrm{WDM})^{3}$. The latter is characterised by solid-like density and temperatures of a few $\mathrm{eV}$, conditions actually found in astrophysical contexts ${ }^{4}$ or in devices for plasma creation and inertial confinement fusion ${ }^{5}$. The advent of ultra-fast/ultra-intense lasers has opened up the possibility to generate and probe such extreme states of matter in transient conditions ${ }^{2,6}$. However, the capability of optical lasers to probe the excited sample is hampered by the "screening effect" of the excited plasma, which drastically limits the penetration length for photons with frequency $(\omega)$ lower than the plasma frequency:

$$
\omega_{p}=\sqrt{N_{e} e^{2} / m_{e} \epsilon_{0}}
$$

where $N_{e}, e, m_{e}$ and $\epsilon_{0}$ are the free electron density, the elementary charge, the effective electron mass and the dielectric constant, respectively ${ }^{3,7}$.

In this context, the development of Free Electron Lasers (FELs) operating in the extreme ultraviolet (XUV) and $\mathrm{x}$-ray spectral ranges represent a landmark result ${ }^{3}$, since the high energy photons emitted by these sources can penetrate matter at solid densities ensuring uniform excitation of the sample. FEL-based experiments have provided valuable new insights into this field, for example saturable absorption by core electrons at XUV/xray wavelengths $s^{8}$ or depression of the ionization potential in solid-density plasma ${ }^{9}$. Other FEL-based experiments aimed at the determination of the relevant parameters of these highly excited states of matter have been proposed, such as $\mathrm{X}$-ray induced changes in optical reflectivity ${ }^{10-12}$, the parallel pyrometrical characterization of heat waves for the determination of the ion temperature $\left(T_{i}\right)^{13}$ or Thompson scattering for the determination of both free electron density $\left(N_{e}\right)$ and temperature $\left(T_{e}\right)^{14}$. The latter approach is based on the measurement of the plasmon lineshape in the inelastic scattering spectra of the excited sample. These highly informative experiments rely on the development of sophisticated XUV/x-ray spectrometers and are limited by coherence issues ${ }^{15}$ and, in high-Z samples, by the low scattering cross-sections ${ }^{14}$.

Information on the plasma resonance (and thus on the related sample parameters) can be also gained by simpler reflectivity measurements ${ }^{16-18}$. For instance, a shift in $\omega_{p}$ upon photo-excitation, observable in Thompson scattering experiments, can be ascribed, through Eq. (1), to a change in $N_{e}$. However, the same shift in $\omega_{p}$ also leads to a change in the XUV reflectivity. This is illustrated in Fig. 1, which reports the XUV reflectivity $(R)$ of a metal at given values of $\omega$ for a typical range in $\omega_{p}$ and $\gamma$, calculated using the Drude model (here $\gamma$ is the inverse 


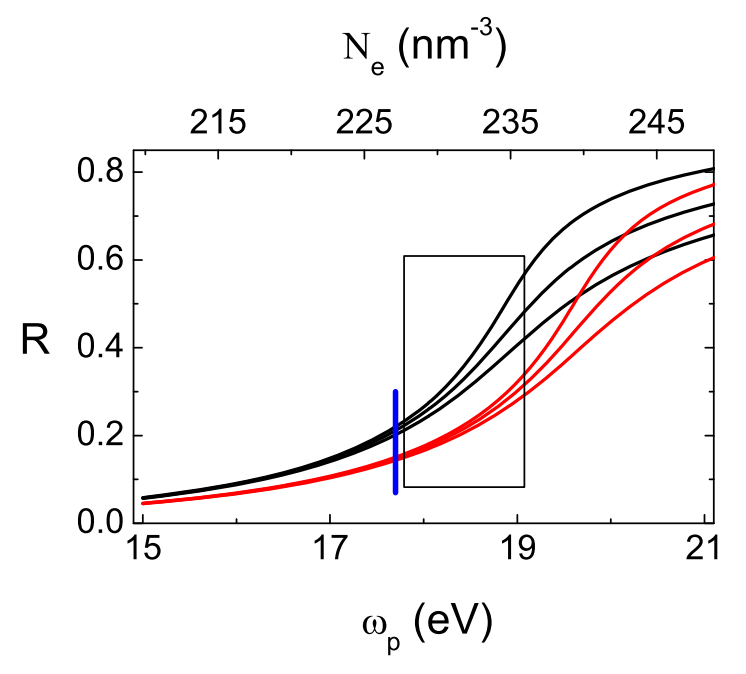

Figure $1 \mid \hbar \omega_{p}$-dependence of $R$ for $\hbar \omega=18.9$ and $20 \mathrm{eV}$ (black/red lines) and $\hbar \gamma=1,1.5$ and $2 \mathrm{eV}$ (higher to lower curves), calculated with Eqs.(3) and (4); the upper horizontal scale is the corresponding range in $N_{e}$ calculated through Eq. (1). The vertical blue segment indicates the expected value of $\hbar \omega_{p}=\hbar \omega_{p}(0)=17.7 \mathrm{eV}$ for the unexcited Ti sample ${ }^{27,28}$, while the hatched area sketches the $\hbar \omega_{p}$-range reached in the excited sample in the exploited $F$-range.

lifetime of the plasma resonance ${ }^{19-21}$, see Methods for further details). If $\omega$ is tuned just above $\omega_{p}$ then the dependence of $R$ on $\omega_{p}$ is more significant than that on $\gamma$. In this case any variation in $R$ can be essentially attributed to a variation in $\omega_{p}$ and thus in $N_{e}$. Following this idea we performed an XUV reflectivity experiment on a Ti sample as a function of both photon fluence $(F)$ and $\omega$. The experiment was carried out at the TIMEX end-station of the Elastic and Inelastic Scattering (EIS) beamline ${ }^{22,23}$, exploiting the broad $\omega$-tunability in the low frequency side of the XUV spectrum provided by the FERMI FEL source ${ }^{24-26}$; experimental details are reported in the Methods section.

\section{Results}

The dependence of $R$ on $F$ is shown in Fig. 2 for three representative values of $\hbar \omega$ and clearly show an increase of reflectivity up to about $40-50 \%$ for $F$-values as large as $20-25 \mathrm{~J} / \mathrm{cm}^{2}$. No appreciable changes in $R$ has been observed for $F<0.02 \mathrm{~J} / \mathrm{cm}^{2}$ (see the Methods section). Assuming that the increase in $N_{e}$ (and thus that in $\omega_{p}$ ) is proportional to the energy density $(\bar{E})$ deposited into the sample close to the metal surface, it is possible to calculate the $F$-dependence of $R$ at a given value of $\omega$. For this purpose we used the Drude model to account for metal reflectivity, in which we consider the time and transverse spatial profiles of the FEL pulse and the fractional increase in $N_{e}$ per unit $\bar{E}$, i.e.:

$$
\omega_{p}^{2}=\omega_{p}^{2}(0)(1+A \bar{E}),
$$

where $\omega_{p}^{2}(0)=17.7 \mathrm{eV}^{27,28}$ is the plasma frequency of the unperturbed sample (more details of the model used for the calculations are reported in the Methods section) and $A$ is an empirical constant. Curves reported in Fig. 2 were obtained using $A=7.5 \cdot 10^{12} \mathrm{~m}^{3} / \mathrm{J}$; higher/lower dashed lines correspond to $A$-value $25 \%$ larger/smaller than $7.5 \cdot 10^{12} \mathrm{~m}^{3} / \mathrm{J}$ and can be regarded as an estimate of the confidence interval (see Supplemental Information), which roughly matches the deviation of the data from the calculated curves.

Once the parameter $A$ is determined, it is straightforward to compute the $F$-dependencies of $\omega_{p}$ and $N_{e}$, and these indicate an increase in $\omega_{p}$ and $N_{e}$ (averaged over both the time and transverse spatial profiles of the FEL pulse) of up to $\approx 4 \%$ and $8 \%$, respectively, at the

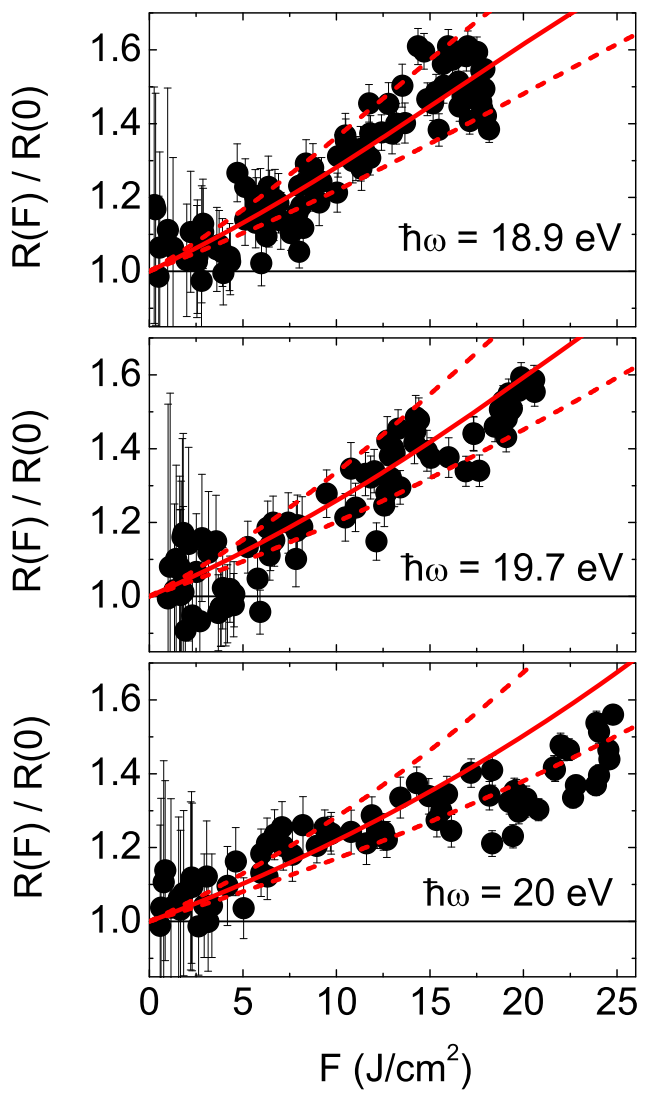

Figure $2 \mid F$-dependence of $R(F) / R(0)$ for some selected $\hbar \omega$-values, indicated in the individual panels. Each data corresponds to a single shot measurement taken in a fresh portion of the sample. Red full lines are the $R(F) / R(0)$ trends calculated through Eq. (6); dashed lines are the estimate of the confidence interval.

highest $F$-values. Fig. 3 reports the variation with $F$ of the mean ion charge: $Z(F)=Z(0) N_{e}(F) / N_{e}(0)$, where $Z(0)=4^{27-29}$. Such a variation is most likely due to the ionization of the shallower $(3 p)$ core electrons, that cannot efficiently recombine within the FEL pulse duration, thus leading to a net increase in the average density of free electrons. We note that the $\bar{E}$-values reached in the present experiment compares well with the ionization energy $\left(E^{+}=99 \mathrm{eV} /\right.$ atom ${ }^{29,30}$ ) for the $\mathrm{Ti}^{4+} \rightarrow \mathrm{Ti}^{5+}$ process. If we assume that the latter is the leading ionization process, then the data shown in Fig. 3 indicates that at the highest probed $F$ s and within the $\approx 45$ fs FEL pulse duration $\approx 40 \%$ of Ti atoms are ionized. This is in qualitative agreement with the results reported in Ref. 8 for $\mathrm{Al}$, where the onset of a

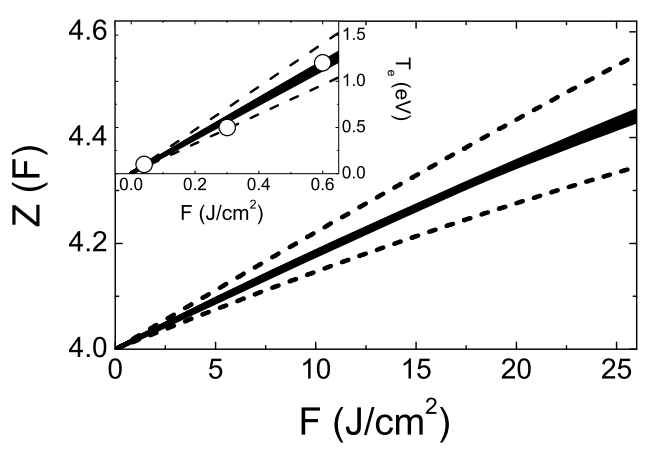

Figure $3 \mid$ Full lines are the F-dependencies of $Z(F)$ at the employed $\hbar \omega$ values; dashed lines are the confidence range. Inset compares the low $F$ estimate of $T_{e}$ with data from Ref. 8 (circles). 


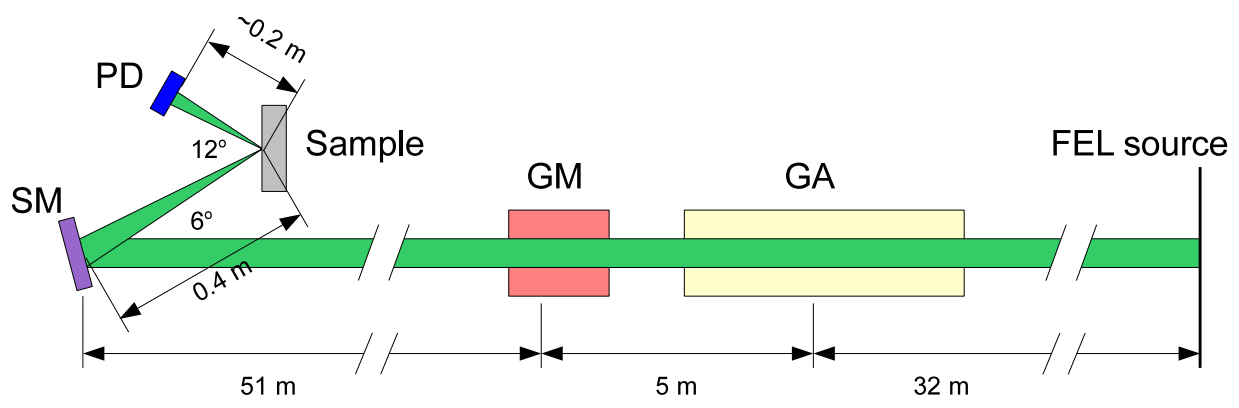

Figure $4 \mid$ Sketch of the experimental setup (side view - not to scale): GA, GM, SM and PD are the gas attenuator cell, the gas monitor, the spherical focusing mirror and the photodiode, respectively. The FEL beam is shown in green. The relevant distances and angles are indicated.

FEL-induced saturable absorption is observed in a similar F-range. This corresponds to the situation where almost all atoms are ionized within the FEL pulse duration. Furthermore, by making the crude assumption that in a moderately excited sample $\bar{E}=(3 / 2) k_{B} T_{e}(F) Z(F)+(Z(F)-Z(0)) E^{+3}$ one can derive $T_{e}$ from $Z$. The $T_{e}$ values estimated in this way are shown in the inset of Fig. 3 and compared with those reported in ref 8 for Al. The latter data were obtained by interpreting the soft $\mathrm{x}$-ray emission spectra within a free electron gas model (like the one used in the present work), so that we can speculatively infer a common low- $F$ behavior of highly photoexcited metals in the sub- 0.1 ps timescale, which is mainly determined by the role played by free electrons.

\section{Discussion}

We have reported the experimental observation of a large XUV reflectivity enhancement in titanium upon irradiation with ultrafast high-fluence FEL-pulses, whose photon frequency was tuned above the plasma frequency of the sample. The FEL irradiation drives the titanium sample into a transient (short-lived) excited state that exhibits electronic temperatures typical of dense plasmas but still retains a cold atomic lattice. Reflectivity data were interpreted through a simple analytical model, based on the free electron gas approach, which employs a single empirical parameter to account for the increase in the plasma frequency within the FEL pulse duration. Such a simplified data analysis is able to capture the main physical process at the base of the observed phenomenology and allows us to determine the mean free electron density of the excited sample.

The information that can be gained from the present experiment is of the highest relevance for the study of highly excited states of matter. Analogous information can be deduced from the lineshape analysis of Thompson scattering spectra. However, the present approach has the advantage of the extreme simplification of the experimental setup and, additionally, reflectivity measurements do not suffer from cross-section or coherence issues. Substantial improvements in the experimental accuracy and data analysis can be achieved with moderate efforts and will likely open up the possibility to obtain additional information from this class of experiments, for example possible modifications of the electronic band structure. Also, the capability of FERMI to operate in the near future at photon energies down to $\approx 12 \mathrm{eV}$ will allow one to probe the condition where the metal reflectivity is almost insensitive to plasma frequency variations but strongly depends on the plasmon lifetime. Since the latter is mostly determined by electron-electron and electron-phonon interactions, information on these dynamics could be gained as well. In summary, the possibility to map out FEL-induced reflectivity variations in a broad range across the low frequency side of the XUV spectrum can be profitably used as a complementary tool with respect to Thompson scattering methods by the growing community involved in the study of matter under extreme conditions.

\section{Methods}

XUV reflectivity measurements. A sketch of the experimental setup is shown in Fig. 4. A Ti mirror (substrate: $\mathrm{Si}$, roughness $\approx 1 \mathrm{~nm}$ RMS, thickness $100 \mathrm{~nm}$, passivated with $3 \mathrm{~nm} \mathrm{TiO}_{2}$ ) was loaded into the 5-axis manipulator (x,y,z,pitch,roll) of the EIS-TIMEX end-station. The FEL beam was focused onto the sample by a spherical platinum-coated silicon mirror ( $400 \mathrm{~mm}$ radius of curvature, $0.2 \mathrm{~nm}$ roughness RMS) placed close to normal incidence (angle of incidence $3^{\circ}$ ), which is able to provide a focal spot of area $\sigma^{2} \approx 100 \mu \mathrm{m}^{2}$. The maximum energy/pulse delivered by the FEL source was in the $\approx 300 \mu \mathrm{J}$ level. The actual energy/pulse at the input of the photon transport system of the EIS-TIMEX beamline $\left(I_{F E L}\right)$ was varied in the $1-300 \mu \mathrm{J}$ range by a gas attenuator and monitored on a shot-to-shot base by a gas monitor $^{31}$. Each shot was tagged by a unique label (bunch number) to one-to-one correlate all the acquired data. $F$ was determined from $I_{F E L}$ taking into account $\sigma^{2}$ as well as the transmission of the $\mathrm{TiO}_{2}$ layer, the beamline transmission and the mirror reflectivity that, in the used $\omega$-range, are in the $\approx 73 \%, 52-56 \%$ and $15-20 \%$ ranges, respectively. Appreciable damage of the sample surface was observed after irradiation with a single FEL pulse with $F>0.1 \mathrm{~J} / \mathrm{cm}^{2}$. The pulse energy reflected by the sample $\left(I_{R}\right)$, at $6^{\circ}$ angle of incidence, was collected at $\hbar \omega=18.9,19.1,19.4,19.7,20.0$ and $20.3 \mathrm{eV}$ by a Silicon photodiode (UVG20S, IRD inc) coupled with a $0.5 \mathrm{~mm}$ thick YAG fluorescence screen having a $100 \mathrm{~nm}$ aluminum coating on the FEL side. In order to calibrate the high- $F$ experiment we collected the ratio $I_{R}^{0} / I_{F E L}^{0}=I_{R} / I_{F E L}$ in the 1-300 $\mu \mathrm{J} I_{F E L}$-range after having moved the sample $3 \mathrm{~mm}$ away from the focal plane (here $F<0.02 \mathrm{~J} / \mathrm{cm}^{2}$ ). The results are shown in Fig. 5 and display a constant ratio, indicating no appreciable changes in the reflectivity in this low- $F$ range. The $I_{R}^{0} / I_{F E L}^{0}$ value was used to determine the relative reflectivity variation in the high- $F$ experiment, i.e.: $R(F) / R(0)=\left(I_{R} / I_{F E L}\right) /\left(I_{R}^{0} / I_{F E L}^{0}\right)$.

Data analysis. The reflection coefficient at near normal incidence can be expressed in terms of the complex dielectric function $(\epsilon) \mathrm{as}^{7}$ :

$$
R \approx\left|\frac{\sqrt{\epsilon}-1}{\sqrt{\epsilon}+1}\right|^{2}
$$

which is a sufficiently accurate approximation for sample thickness much larger than the penetration depth of the radiation $(L<23 \mathrm{~nm}$ in the present case). In metals a simple expression for $\epsilon$ is given by the Drude formula ${ }^{7}$ :

$$
\epsilon=1-\frac{\omega_{p}^{2}}{\omega^{2}+i \omega \gamma} .
$$

In this model, a variation in $R$ is ascribed to a change in $\omega_{p}$ or $\gamma$ when the photon pulse impinges. However, as shown in Fig. 1, in the used $\omega$ range the dependence on $\omega_{p}$ is much more significant than on $\gamma$. So we arbitrarily set $\hbar \gamma=1.5 \mathrm{eV}$, in analogy with

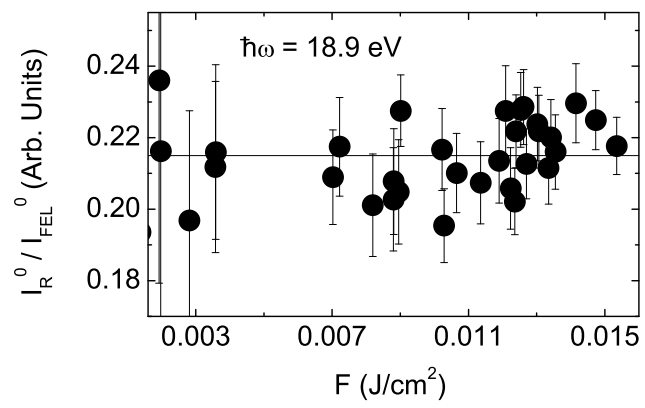

Figure $5 \mid I_{R}^{0} / I_{F E L}^{0}$-values as a function of $F$ in the low- $F$ range for $\hbar \omega=$ $18.9 \mathrm{eV}$. 


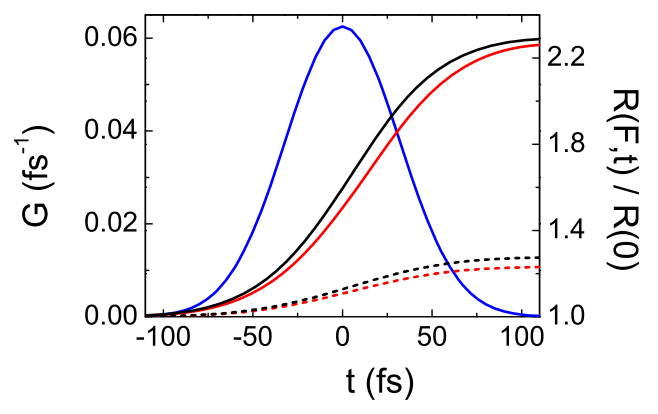

Figure $6 \mid t$-dependence of $G(t)$ (left scale; blue line) and of $R(F, t) / R(0)$ (right scale; black/red and full/dashed lines are $\hbar \omega=18.9 / 20 \mathrm{eV}$ and $F=$ $20 / 5 \mathrm{~J} / \mathrm{cm}^{2}$, respectively).

what was found for $\mathrm{Al}$ (where $\left.\gamma \approx 0.1 \cdot \omega_{p}(0)^{19}\right)$ since a reference value for $\mathrm{Ti}$ is not available in the literature, and we drop a possible $F$-dependence of $\gamma$. We also stress that within the accuracy of the present experiment the results are only marginally affected by the choice of the $\hbar \gamma$-value within the $0.5-2.5 \mathrm{eV}$ range, which essentially includes the $\hbar \gamma$-values observed in a large number of metals ${ }^{19-21}$, as also shown in the Supplemental Information. For this reason the observed increase in $R$ was related to an increase in $\omega_{p}$ and then in $N_{e}$ through Eq. (1). As quoted in the main text, in order to provide a quantitative estimate we assumed that $N_{e}$ is proportional to $\bar{E}$ and we considered both the time $(t)$ and transverse spatial $(x, y)$ profiles of the FEL pulse. These result in a $(t, x, y)$-evolution of the reflectivity $(R(F, t, x, y))$, which can be calculated using Eqs. (3) and (4) assuming $\omega_{p}^{2}(F, t, x, y)=\omega_{p}^{2}(0)(1+A \bar{E}(F, t, x, y))$, where

$$
\bar{E}(F, t, x, y)=\frac{F \sigma^{2}}{L} \int_{-\infty}^{t} d t^{\prime} S(x, y) G\left(t^{\prime}\right)\left(1-R\left(F, t^{\prime}, x, y\right)\right)
$$

is the spatial profile of the energy density at time $t$ deposited in a sample layer (involved in the reflectivity process) of thickness $d<L$ below the surface, where $G(t)$ and $S(x, y)$ are the normalized time and space intensity distributions. These are approximated by Gaussian functions with $\sigma_{x}=\sigma_{y}=5 \mu \mathrm{m}$ and $\sigma_{t}=45$ fs. $\sigma_{t}$ was evaluated as $\sigma_{t}=\sigma_{\text {seed }} / \sqrt[3]{N^{32}}$, where $\sigma_{\text {seed }}=70 f s$ is the time duration of the seed laser pulse (measured with an optical cross-correlator) and $N=4$ is the harmonic number at which the FEL radiators were tuned. Representative $t$-traces of $R(F, t) / R(0)=(1 /$ $R(0)) \int d x \int d y S(x, y) R(F, t, x, y)$ are shown in Fig. 6 along with $G(t)$. It is worth stressing that the possibility to approximate $G(t)$ with a well defined function (which ultimately permitted us to carry out the present data analysis) is one of the advantages related to the use of seeded FELs. Finally, the factor $1-R(F, t, x, y)$ in Eq. (5) accounts for the reduction in the amount of energy available at later times for further excitation due to the $t$-increase in $\omega_{p}$ (and thus in $R$ ) within the FEL pulse duration. Finally, the observed $R(F) / R(0)$ is simply given by the space-time average of $R(F, t, x, y)$, i.e.:

$$
\frac{R(F)}{R(0)}=\frac{1}{R(0)} \int d x \int d y \int d t S(x, y) G(t) R(F, t, x, y) .
$$

The mean ion charge (or equivalently the average free electron density) can be calculated as well through the definition of $\omega_{p}$ (see Eq. (1)), i.e.:

$$
Z(F)=\frac{N_{e}(F)}{N_{a}}=\frac{Z(0)}{\omega_{p}^{2}(0)} \int d x \int d y \int d t S(x, y) G(t) \omega_{p}^{2}(F, t, x, y) .
$$

1. Holzapfel, W. B. Physics of solids under strong compression. Rep. Prog. Phys. 59, 29-90 (1996).

2. Ross, M. Matter under extreme conditions of temperature and pressure. Rep. Prog. Phys. 48, 1-52 (1985).

3. Lee, R. W. et al. Finite temperature dense matter studies on next-generation light sources. J. Opt. Soc. Am. B 20, 770-778 (2003).

4. Taylor, R. J. The Stars: their structure and evolution (Cambridge University Press, Cambridge, 1994).

5. Hu, S. X., Militzer, B., Goncharov, V. N. \& Skupsky, S. Strong coupling and degeneracy effects in inertial confinement fusion implosions. Phys. Rev. Lett. 104, 235003 (2010).

6. Ping, Y. et al. Broadband dielectric function of nonequilibrium warm dense gold. Phys. Rev. Lett. 96, 255003 (2006).

7. Wooten, F. Optical Properties of Solids (Academic Press, New York, 1972).

8. Nagler, B. et al. Turning solid aluminium transparent by intense soft X-ray photoionization. Nat. Phys. 5, 693-696 (2009).

9. Ciricosta, O. et al. Direct measurements of the ionization potential depression in a dense plasma. Phys. Rev. Lett. 109, 065002 (2012).

10. Durbin, S. M. X-ray induced optical reflectivity. AIP Advances 2, 042151 (2012).
11. Gahl, O. et al. A femtosecond X-ray/optical cross-correlator. Nat. Photonics 2, 165-169 (2008).

12. Krupin, O. et al. Temporal cross-correlation of $\mathrm{x}$-ray free electron and optical lasers using soft $\mathrm{x}$-ray pulse induced transient reflectivity. Opt. Express 20, 11396-11406 (2012).

13. Principi, E. et al. Determination of the ion temperature in a stainless steel slab exposed to intense ultrashort laser pulses. Phys. Rev. Lett. 109, 025005 (2012).

14. Gregori, G., Glenzer, S. H., Rozmus, W., Lee, R. W. \& Landen, O. L. Theoretical model of $\mathrm{x}$-ray scattering as a dense matter probe. Phys. Rev. E 67, 026412 (2003).

15. Gregori, G., Tommasini, R., Landen, O. L., Lee, R. W. \& Glenzer, S. H. Limits on collective X-ray scattering imposed by coherence. Europhys. Lett. 74, 637-643 (2006).

16. Moss, T. S., Hawkins, T. D. F. \& Burrell, G. J. Use of plasma edge reflection measurements in the study of semiconductors. J. Phys. C: Solid State Phys. 1, 1435-1446 (1968).

17. Shank, C. V., Yen, R. \& Hirlimann, C. Time-resolved reflectivity measurements of femtosecond-optical-pulse-induced phase transitions in silicon. Phys. Rev. Lett. 50, 454-457 (1983)

18. Mao, H. K., Hemley, R. J. \& Hanfland, M. Infrared reflectance measurements of the insulator-metal transition in solid hydrogen. Phys. Rev. Lett. 65, 484-487 (1990).

19. Ujihara, K. Reflectivity of metals at high temperatures. J. Appl. Phys. 43, 2376-2383 (1972).

20. Sturm, K. \& Oliveira, L. E. Wave-vector-dependent plasmon linewidth in the alkali metals. Phys. Rev. B 24, 3054-3062 (1981).

21. Chen, C. H., Joy, D. C., Chen, H. S. \& Hauser, J. J. Observation of anomalous plasmon linewidth in the icosahedral Al-Mn quasicrystals. Phys. Rev. Lett. 57, 743-746 (1986)

22. Di Cicco, A. et al. Probing matter under extreme conditions at FERMI@Elettra: the TIMEX beamline Proc. of SPIE 8077, 807704 (2011).

23. Bencivenga, F. \& Masciovecchio, C. FEL-based transient grating spectroscopy to investigate nanoscale dynamics. Nucl. Instrum. Meth. Phys. Res. A 606, 785-789 (2009).

24. Allaria, E. et al. The FERMI@Elettra free-electron-laser source for coherent x-ray physics: photon properties, beam transport system and applications New. J. Phys. 12, 075002 (2010).

25. Allaria, E. et al. Highly coherent and stable pulses from the FERMI seeded freeelectron laser in the extreme ultraviolet. Nat. Photonics 6, 699-704 (2012).

26. Allaria, E. et al. Tunability experiments at the FERMI@Elettra free-electron laser New. J. Phys. 14, 113009 (2012).

27. Robins, J. L. \& Swan, J. B. Characteristic electron energy loss spectra of the transition metals, Ti to Cu. Proc. Phys. Soc. 76, 857-869 (1960).

28. Bracconi, P. \& Lässer, R. Investigation of titanium and titanium hydride by AES and EELS. Appl. Surf. Science 28, 204-214 (1987).

29. Stambulchik, E. et al. Progress in line-shape modeling of K-shell transitions in warm dense titanium plasmas. J. Phys. A: Math. Theor. 42, 214056 (2009).

30. James, A. M. \& Lord, M. P. Macmillan's Chemical and Physical Data (Macmillan, London, 1992).

31. Zangrando, M. et al. PADReS: the photon analysis delivery and reduction system at the FERMI@Elettra FEL user facility. Rev. Scient. Instrum. 80, 113110 (2009).

32. Ratner, D., Fry, A., Stupakov, G. \& White, W. Laser phase errors in seeded free electron lasers. Phys. Rev. ST Accel. Beams 15, 030702 (2012).

\section{Acknowledgments}

The authors acknowledge the invaluable support provided by the whole FERMI team and $\mathrm{K}$. C. Prince for proof reading of the manuscript. C. Masciovecchio acknowledges support from the European Research Council through the ERC Grant N.202804-TIMER. A. Di Cicco acknowledges support from Elettra-Sincrotrone Trieste (ST) through the TIMEX collaboration.

\section{Author contributions}

F.B. conceived the experiment and data interpretation. E.P., E.G. and A.G. realized the experimental setup. F.B., E.P., E.G., R.C., A.B., F.D.A., A.D.C., S.D.F., M.M., L.P. and M.S performed the measurements. F.B. carried out the data analysis. F.B., C.M., E.G., E.P., A.D.C., R.G. and A.F. discussed the data and wrote the paper.

\section{Additional information}

Supplementary information accompanies this paper at http://www.nature.com/ scientificreports

Competing financial interests: The authors declare no competing financial interests. How to cite this article: Bencivenga, F. et al. Reflectivity enhancement in titanium by ultrafast XUV irradiation. Sci. Rep. 4, 4952; DOI:10.1038/srep04952 (2014).

This work is licensed under a Creative Commons Attribution-NonCommercialShareAlike 3.0 Unported License. The images in this article are included in the article's Creative Commons license, unless indicated otherwise in the image credit; if the image is not included under the Creative Commons license, users will need to obtain permission from the license holder in order to reproduce the image. To view a copy of this license, visit http://creativecommons.org/licenses/by-nc-sa/3.0/ 